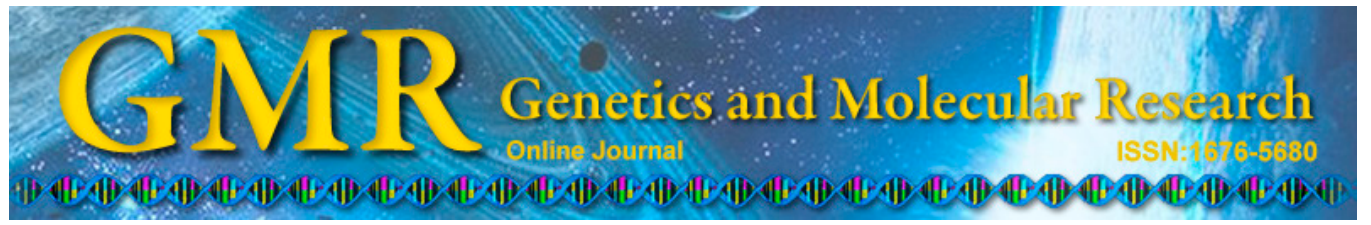

\title{
Influence of interleukin-17 gene polymorphisms on the development of pulmonary tuberculosis
}

\author{
G.-C. Shi and L.-G. Zhang \\ Department of Tuberculosis, \\ The First Affiliated Hospital of Xinxiang Medical University, Weihui, China \\ Corresponding author: G.-C. Shi \\ E-mail: shigc_xxm@163.com
}

Genet. Mol. Res. 14 (3): 8526-8531 (2015)

Received January 8, 2015

Accepted April 28, 2015

Published July 28, 2015

DOI http://dx.doi.org/10.4238/2015.July.28.22

\begin{abstract}
We conducted a case-control study in a Chinese population to examine the correlations between interleukin (IL)-17 gene polymorphisms and tuberculosis (TB) development. The study population included $336 \mathrm{~TB}$ subjects and 351 control subjects who were enrolled between June 2012 and June 2014. Genotyping analyses of IL-17A rs2275913 and rs3748067 and IL-17F rs763780 were analyzed using polymerase chain reaction-restriction fragment length of polymorphism. The genotype distributions of IL-17 rs2275913 were found to be in Hardy-Weinberg equilibrium in the controls, while the IL17 rs3748067 and rs763780 were not. Based on unconditional logistic regression, individuals carrying the AA genotype and GA + AA genotype of rs2275913 were more likely to have a significantly increased risk of TB compared to subjects with the GG genotype. The ORs $(95 \% \mathrm{CI})$ for the AA genotype and GA + AA genotype were 2.20 (1.35-3.60) and 1.52 (1.11-2.09), respectively. The CC genotype and TC + CC genotype of rs763780 were associated with increased risk of TB when compared with the TT genotype. The ORs $(95 \% \mathrm{CI})$ for the CC genotype and TC + CC genotype were 1.99 (1.05-3.87) and 1.58 (1.07-2.33), respectively. In conclusion, rs763780 may play a critical role in the etiology of TB.
\end{abstract}

Key words: Interleukin-17; Polymorphism; Pulmonary tuberculosis 


\section{INTRODUCION}

Tuberculosis (TB) is an infectious disease with the highest death rate worldwide, despite the fact that the first anti-tuberculosis drug was introduced approximately 50 years ago (Lawn and Zumla, 2011). It is estimated that 8.1 million people were diagnosed with active TB and 1.3 million died from it by the World Health Organization in 2011 (Lawn and Zumla, 2011). It is reported that about $60 \%$ of the world's population have been infected with pathogen mycobacterium tuberculosis, but less than $10 \%$ of these TB-infected cases will present clinical disease during their lifetimes.

Previous studies have reported that cytokine genes contribute to susceptibility of TB, such as interferon-1 $\beta$, interleukin (IL)-3, IL-6, IL-10, and IL-12B IL-17 as well as IL-18 (Selvaraj et al., 2008; Abhimanyu et al., 2011; Meenakshi et al., 2013; Feng et al., 2014; Tiwari et al., 2014; Zhang et al., 2014). However, the results have been inconsistent.

Th17 cells are a unique subset of effector T helper cells that subvert the Th1 and Th2 lineages (Harrington et al., 2005). Increasing studies have reported that Th17 cells are the main IL-17-producing cells and contribute to protective immunity against Mycobacterium tuberculosis (Khader et al., 2007; Chen et al., 2009; Paidipally et al., 2009). Khader et al. (2007) reported that Th17 response had a role in protective immunity in M. tuberculosis infection, and M. tuberculosis infection was correlated with a decreased Th17 response because of suppressing Th1 cytokines (Khader et al., 2007). Therefore, Th17 cells not only contribute to the development of M. tuberculosis, but also protect against intracellular pathogens (Pitta et al., 2009).

Previous studies reported an association between genetic polymorphisms in IL-17 and the susecptibility of TB (Abhimanyu et al., 2013; Ocejo-Vinyals et al., 2013; Peng et al., 2013; Tiwari et al., 2014), but the results of previous studies are inconsistent. Therefore, we conducted a case-control study in a Chinese population to examine the correlations between IL-17 gene polymorphisms and TB development.

\section{MATERIAL AND METHODS}

\section{Study population}

A total of 336 TB subjects and 351 control subjects were collected from First Affiliated Hospital of Xinxiang Medical University between June 2012 and June 2014. TB patients were diagnosed based on X-rays and bacteriologically with both positive sputum smear examination and culture for M. tuberculosis. The excluded criteria for TB cases were those with extra pulmonary TB in organs other than the lungs, and with human immunodeficiency virus-positive. A total of 351 control subjects who had undergone routine healthy examination in the First Affiliated Hospital of Xinxiang Medical University during the same period. The inclusion criteria for the control subjects were free of TB and had no history of an inflammatory autoimmune disease.

A detained questionnaire was taken to collect the demographic information of TB patients and controls, including age, gender, tobacco smoking and severity levels. A written informed consent form was completed by all TB patients and controls before participating in the study. Our study protocol was approved by the ethics committee of the First Affiliated Hospital of Xinxiang Medical University.

\section{Single nucleotide polymorphism selection and genotyping analysis}

Three single nucleotide polymorphisms of IL-17A rs2275913 and rs3748067 and IL-17F 
rs763780 were selected. Each TB patient and control subject provided $5 \mathrm{~mL}$ peripheral blood and stored at $-20^{\circ} \mathrm{C}$ until use. Genomic DNA of IL-17A rs2275913 and rs3748067 and IL-17F rs763780 was isolated from the ethylenediaminetetraacetic acid (EDTA)-anticoagulated peripheral blood samples using a TIANamp blood DNA kit (Tiangen Biotech, Beijing, China) according to the manufacturer instructions. Genotyping analyses of IL-17A rs2275913 and rs3748067 and IL-17F rs763780 were analyzed using polymerase chain reaction-restriction fragment length of polymorphism. The positive and reverse primers of IL-17A rs2275913 and rs3748067 and IL-17F rs763780 were designed using the Primer 5.0 software (Premier Biosoft, Palo Alto, CA, USA). Polymerase chain reaction was performed using the following conditions: initial denaturation at $94^{\circ} \mathrm{C}$ for $5 \mathrm{~min}$, followed by 35 cycles of denaturation at $94^{\circ} \mathrm{C}$ for $45 \mathrm{~s}$, annealing at $62^{\circ} \mathrm{C}$ for 60 $\mathrm{s}$ and extension at $72^{\circ} \mathrm{C}$ for $60 \mathrm{~s}$, and final extension at $72^{\circ} \mathrm{C}$ for $10 \mathrm{~min}$. The PCR products were visualized by $1.0 \%$ agarose gel electrophoresis with ethidium bromide staining and UV light.

\section{Statistical analysis}

Continuous variables are reported as means \pm standard deviation (SD), and categorical variables were expressed as frequencies and percentage (\%). Student $t$-test or $\chi^{2}$-test was used to compare continuous variables and categorical variables between case and control groups. Hardy-Weinberg equilibrium for IL-17A rs2275913 and rs3748067 and IL-17F rs763780 among controls was compared using chi-squared goodness of fit test. Unconditional logistic regression analysis was performed to evaluate the effects of IL-17A rs2275913 and rs 3748067 and IL-17F rs 763780 polymorphisms on the risk of TB, with results expressed as odds ratios (ORs) and corresponding 95\% CI. Homozygotes of the most frequent genotype were considered as the reference group for analysis. For all statistical analyses, values were considered to be statistically significant when $\mathrm{P}<0.05$.

\section{RESULTS}

This study included $336 \mathrm{~TB}$ cases (70.54\% males and $29.46 \%$ females) and 351 controls (69.52\% males and $30.48 \%$ females) (Table 1). The mean ages for TB cases and controls were 42.5 \pm 10.6 and $41.3 \pm 10.4$ years, respectively. There were no significant differences in the distribution of gender, age, and tobacco smoking between TB cases and controls. Two hundred and seventy TB cases $(80.36 \%)$ were mild-moderate level of TB and 66 (19.64\%) were advanced level of TB.

\begin{tabular}{|c|c|c|c|c|c|c|}
\hline Variables & Cases & $\%$ & Control & $\%$ & $\chi^{2}$ value & $P$ value \\
\hline \multicolumn{7}{|l|}{ Age, years } \\
\hline$<50$ & 240 & 71.43 & 243 & 69.23 & & \\
\hline$\geq 50$ & 96 & 28.57 & 108 & 30.77 & 0.39 & 0.52 \\
\hline \multicolumn{7}{|l|}{ Gender } \\
\hline Female & 99 & 29.46 & 107 & 30.48 & & \\
\hline Male & 237 & 70.54 & 244 & 69.52 & 0.09 & 0.77 \\
\hline \multicolumn{7}{|l|}{ Tobacco smoking } \\
\hline Never & 197 & 58.63 & 229 & 65.24 & & \\
\hline Ever & 139 & 41.37 & 122 & 34.76 & 3.19 & 0.07 \\
\hline \multicolumn{7}{|l|}{ Severity levels } \\
\hline Mild-moderate & 270 & 80.36 & & & & \\
\hline Advanced & 66 & 19.64 & & & & \\
\hline
\end{tabular}


The genotype distributions of IL-17A rs2275913 and rs3748067 and IL-17F rs763780 were shown in Table 2 . By $\chi^{2}$-test, there were no significant differences in the genotype distributions of IL-17 rs2275913, rs3748067, and rs763780 between TB cases and controls. The genotype distributions of IL-17 rs2275913 were found to be in Hardy-Weinberg equilibrium in the controls, while the IL-17 rs3748067 and rs763780 were not. Minor allele frequencies in controls of the 3 single nucleotide polymorphisms in IL-17 were similar to the minor allele frequencies in NCBI.

\begin{tabular}{|c|c|c|c|c|c|c|c|c|c|}
\hline$\overline{\mathrm{SNP}}$ & Cases & $\%$ & Controls & $\%$ & $\chi^{2}$ value & P value & $\begin{array}{c}\text { Minor allele } \\
\text { frequency in NCBI }\end{array}$ & $\begin{array}{c}\text { Minor allele } \\
\text { frequency in controls }\end{array}$ & $\begin{array}{l}\text { P value for Hardy- } \\
\text { Weinberg Equilibrium }\end{array}$ \\
\hline \multicolumn{10}{|c|}{ rs2275913 } \\
\hline GG & 129 & 38.39 & 171 & 48.72 & & & & & \\
\hline GA & 143 & 42.56 & 142 & 40.46 & & & & & \\
\hline AA & 64 & 19.05 & 38 & 10.83 & 12.19 & 0.002 & 0.2927 & 0.3105 & 0.30 \\
\hline \multicolumn{10}{|c|}{ rs3748067 } \\
\hline $\mathrm{CC}$ & 277 & 82.44 & 303 & 86.33 & & & & & \\
\hline CT & 40 & 11.90 & 37 & 10.54 & & & & & \\
\hline TT & 19 & 5.66 & 11 & 3.13 & 3.09 & 0.21 & 0.0769 & 0.0840 & $<0.001$ \\
\hline \multicolumn{10}{|c|}{ rs763780 } \\
\hline $\mathrm{TT}$ & 250 & 74.40 & 289 & 82.34 & & & & & \\
\hline $\mathrm{TC}$ & 54 & 16.07 & 44 & 12.54 & & & & & \\
\hline $\mathrm{CC}$ & 31 & 9.23 & 18 & 5.13 & 6.92 & 0.03 & 0.0935 & 0.1766 & $<0.001$ \\
\hline
\end{tabular}

The associations between the IL-17A rs2275913 and rs3748067 and IL-17F rs763780 polymorphisms and risk of TB are shown in Table 3. Based on unconditional logistic regression, individuals carrying the AA genotype and GA + AA genotype of rs2275913 were more likely to have a significantly increased risk of TB when compared with the GG genotype. The odds ratio (95\% confidence interval) for the AA genotype and GA + AA genotype of rs 2275913 were 2.20 (1.35-3.60) and 1.52 (1.11-2.09), respectively. Moreover, the CC genotype and TC + CC genotype of rs763780 were associated with increased risk of TB when compared with the TT genotype. The odds ratio (95\% confidence interval) for the CC genotype and TC + CC genotype were 1.99 (1.05-3.87) and 1.58 (1.07-2.33), respectively. However, we found no significant association between rs3748067 and TB development.

Table 3. Association of IL-17 rs2275913, rs3748067 and rs763780 polymorphisms with risk of TB.

\begin{tabular}{lrrrrrr}
\hline Variables & Cases & $\%$ & Control & $\%$ & OR $(95 \% \text { CI })^{1}$ & P value \\
\hline rs2275913 & 129 & 38.39 & 171 & 48.72 & Ref. & \\
GG & 143 & 42.56 & 142 & 40.46 & $1.33(0.95-1.87)$ & 0.08 \\
GA & 64 & 19.05 & 38 & 10.83 & $2.20(1.35-3.60)$ & $<0.001$ \\
AA & 207 & 61.61 & 180 & 51.29 & $1.52(1.11-2.09)$ & 0.006 \\
GA+ AA & & & & & Ref. & \\
rs3748067 & 277 & 82.44 & 303 & 86.33 & $1.18(0.71-1.96)$ & 0.49 \\
CC & 40 & 11.90 & 37 & 10.54 & $1.89(0.84-4.47)$ & 0.1 \\
CT & 19 & 5.66 & 11 & 3.13 & & \\
TT & 59 & 17.56 & 48 & 13.67 & $1.34(0.87-2.08)$ & \\
CT + TT & & & & & Ref. & 0.11 \\
rs763780 & 250 & 74.40 & 289 & 82.34 & $1.42(0.90-2.24)$ & 0.02 \\
TT & 54 & 16.07 & 44 & 12.54 & $1.99(1.05-3.87)$ & 0.01 \\
TC & 31 & 9.23 & 18 & 5.13 & & $1.58(1.07-2.33)$ \\
CC & 85 & 25.30 & 62 & 17.67 & & \\
TC + CC & &
\end{tabular}

${ }^{1}$ Adjusted for gender, age, tobacco smoking, and alcohol consumption. 


\section{DISCUSSION}

In this study, we conducted a case-control study to evaluate the association between IL-17A rs2275913 and rs3748067 and IL-17F rs763780 polymorphisms and risk of TB in a Chinese population. We observed that individuals carrying the AA genotype and the GA + AA genotype of rs2275913 and the CC genotype and the TC + CC genotype of rs 763780 were associated with increased risk of TB compared with the wide-type genotype, suggesting that IL-17 gene polymorphisms contribute to the susceptibility of TB.

Multiple previous studies have reported an association between IL-17 gene polymorphisms and autoimmune diseases (Jin et al., 2011; Zhang et al., 2013; Maalmi et al., 2014; Shen et al., 2015). Jin et al. (2011) reported an association between IL-17 gene polymorphisms and susceptibility to asthma in a Korean population, and suggested that the rs1889570 polymorphism was correlated with the development of asthma. Shen et al. (2015) reported that the IL-17 rs2275913 and rs3819024 variant alleles were associated with a decreased risk of rheumatoid arthritis, while the IL-17 rs3819025 and rs8193036 variant alleles were correlated with an increased risk of rheumatoid arthritis. Maalmi et al. (2014) conducted a study in a Tunisian population, and found that the IL-17A rs 2275913 and IL-17F rs 763780 were associated with an increased risk of asthma in children. Zhang et al. (2013) examined the association between IL-17 gene polymorphisms and inflammatory bowel disease, and reported that the IL-17 rs2275913 and rs763780 polymorphisms were associated with an increased risk of inflammatory bowel disease.

For the association between IL-17A and IL-17F gene polymorphisms and TB risk, 4 studies have reported an association, but the results of them were inconsistent (Abhimanyu et al., 2013; Ocejo-Vinyals et al., 2013; Peng et al., 2013; Tiwari et al., 2014). Ocejo-Vinyals et al. (2013) investigated an association between IL-17 rs2275913 polymorphism and susceptibility to TB, and suggested that the GG genotype of IL-17A rs2275913 was associated with an increased risk of pulmonary tuberculosis. Peng et al. (2013) conducted a study of a Chinese population and found that patients who carried the T allele of IL-17 rs763780 were more susceptible to TB compared to $\mathrm{CC}$ genotype, but no significant association was found between rs2275913 polymorphisms and TB risk. However, Tiwari et al. (2014) reported that the IL17 gene polymorphisms were not associated with the development of TB. Abhimanyu et al. (2013) reported no association between IL-17A and IL-17F polymorphisms and risk of TB. In our study, we found that the CC genotype of rs 763780 gene polymorphism was associated with an increased risk of TB. The discrepancies between these results may have been caused by differences in ethnicities, study design, and sample sizes.

There were three limitations to this study. First, genotype distributions of IL-17 rs3748067 and rs763780 were not in Hardy-Weinberg equilibrium in the control group. These results suggested that the controls did not represent the distribution of the general population. Therefore, some selection bias may have existed in this study. Second, the small sample size of this study may have limited the statistical power of identifying the difference between groups. Third, other genetic polymorphisms may influence the risk of TB, except for the inflammatory cytokines. Therefore, further large-sample studies are needed to investigate the association between inflammatory cytokines and susceptibility of TB.

In summary, we suggest that the AA genotype and GA + AA genotype of rs2275913 and the CC genotype and TC + CC genotype of rs763780 polymorphisms were associated 
with an increased risk of TB, and rs2275913 and rs763780 contribute to the etiology of TB. Further large-sample studies are greatly needed to confirm these associations.

\section{REFERENCES}

Abhimanyu, Mangangcha IR, Jha P, Arora K, et al. (2011). Differential serum cytokine levels are associated with cytokine gene polymorphisms in north Indians with active pulmonary tuberculosis. Infect. Genet. Evol. 11: 1015-1022.

Abhimanyu, Bose M, Komal, Varma-Basil M (2013). Lack of association between IL17A and IL17F polymorphisms and related serum levels in north Indians with tuberculosis. Gene 529:195-198.

Chen X, Zhang M, Zhu X, Deng Q, et al. (2009). Engagement of Toll-like receptor 2 on CD4(+) T cells facilitates local immune responses in patients with tuberculous pleurisy. J. Infect. Dis. 200: 399-408.

Feng FM, Liu XX, Sun YH, Zhang P, et al. (2014). Independent and joint effects of the IL-6 and IL-10 gene polymorphisms in pulmonary tuberculosis among the Chinese Han population. Genet. Mol. Res. 13: 7766-7772.

Harrington LE, Hatton RD, Mangan PR, Turner H, et al. (2005). Interleukin 17-producing CD4+ effector T cells develop via a lineage distinct from the T helper type 1 and 2 lineages. Nat. Immunol. 6: 1123-1132.

Jin EH, Choi EY, Yang JY, Chung HT, et al. (2011). Significant association between IL-17F promoter region polymorphism and susceptibility to asthma in a Korean population. Int. Arch. Allergy Immunol. 155: 106-110.

Khader SA, Bell GK, Pearl JE, Fountain JJ, et al. (2007). IL-23 and IL-17 in the establishment of protective pulmonary CD4+ T cell responses after vaccination and during Mycobacterium tuberculosis challenge. Nat. Immunol. 8: 369-377.

Lawn SD and Zumla AI (2011). Tuberculosis. Lancet 378: 57-72.

Liang B, Guo Y, Li Y, Kong H (2014). Association between IL-10 gene polymorphisms and susceptibility of tuberculosis: evidence based on a meta-analysis. PLoS One 9: e88448.

Maalmi H, Beraies A, Charad R, Ammar J, et al. (2014). IL-17A and IL-17F genes variants and susceptibility to childhood asthma in Tunisia. J. Asthma 51: 348-354.

Meenakshi P, Ramya S, Shruthi T, Lavanya J, et al (2013). Association of IL-1 $\beta+3954$ C/T and IL-10-1082 G/A cytokine gene polymorphisms with susceptibility to tuberculosis. Scand. J. Immunol. 78: 92-97.

Ocejo-Vinyals JG, de Mateo EP, Hoz MÁ, Arroyo JL, et al. (2013). The IL-17 G-152A single nucleotide polymorphism is associated with pulmonary tuberculosis in northern Spain. Cytokine 64: 58-61.

Paidipally P, Periasamy S, Barnes PF, Dhiman R, et al. (2009). NKG2D-dependent IL-17 production by human T cells in response to an intracellular pathogen. J. Immunol. 183: 1940-1945.

Peng R, Yue J, Han M, Zhao Y, Liu L, Liang L (2013). The IL-17F sequence variant is associated with susceptibility to tuberculosis. Gene 515: 229-232.

Pitta MG, Romano A, Cabantous S, Henri S, et al. (2009). IL-17 and IL-22 are associated with protection against human kala azar caused by Leishmania donovani. J. Clin. Invest. 119: 2379-2387.

Selvaraj P, Alagarasu K, Harishankar M, Vidyarani M, et al. (2008). Cytokine gene polymorphisms and cytokine levels in pulmonary tuberculosis. Cytokine 43: 26-33.

Shen L, Zhang H, Yan T, Zhou G, et al. (2015). Association between interleukin 17A polymorphisms and susceptibility to rheumatoid arthritis in a Chinese population. Gene S0378-1119(15)00437-0.

Tiwari U, Ramachandran VG, Das S and Kumar S (2014). Interleukin-3 and interleukin-17 do not play a dynamic role in the immunopathogenesis of osteoarticular tuberculosis. Indian J. Tuberc. 61: 142-147.

Zhang J, Zheng L, Zhu D, An H, et al (2014). Polymorphisms in the interleukin 18 receptor 1 gene and tuberculosis susceptibility among Chinese. PLoS One 9:e110734.

Zhang X, Yu P, Wang Y, Jiang W, et al (2013). Genetic polymorphisms of interleukin 17A and interleukin 17F and their association with inflammatory bowel disease in a Chinese Han population. Inflamm. Res. 62:743-750. 